\title{
CORRIENTES BAROCLÍNICAS Y SECAS INTERNAS EN EL LAGO DE BANYOLES (GIRONA, ESPAÑA)
}

\author{
E. Roget, X. Casamitjana y J. Pararols
}

Institut d'Ecologia Aquàtica (E.G.G.) y Departament de Física, Universitat Autònoma de Barcelona. 17071 Girona. España.

Palabras clave: secas internas, corrientes baroclínicas, lago de Banyoles

\begin{abstract}
BAROCLYNIC CURRENTS AND INTEKNAL SEICHES IN BANYOLES LAKE (SPAIN)

Wind induced phenomena as the set-up of the therrnocline and the consequent internal seiches have been recorded in the southern lobe of Banyoles Lake. The observed period is in agreement with that obtained theoretically by the Mortimer/Defant method. During these events - about two days - the thermocline migrated more than two meters. The Richardson number during this period of seiches suggests that the induced shear was not the force but the direct effect of the wind.

From November to April a bottom baroclinic current from north to south was also recordered. The differential heating between the two lobes is due to their significantly different depth and to the different incoming heat flux through the underground sources. This current can be observed indirectly from temperature data but also directly from the data recordered in a currentmeter too. The current is highly influenced by the wind blowing in the northsouth direction. In the central part of the lake, currents up to $12 \mathrm{~cm} / \mathrm{s}$ are observed. Their path, slightly inflected towards the right, seerns to be influenced by the bottom of the lake, but Coriolis force may be also important.
\end{abstract}

\section{INTRODUCCIÓN}

Los fenómenos característicos de la limnología física han sido escasamente descritos en el caso de lagos pequeños y medianos españoles. En este trabajo se exponen algunas evidencias experimentales de tales procesos, observados en el lago de Banyoles.

Los procesos físicos observados han sido los afloramientos del agua hipolimnética, producidos por efecto del viento y las consiguientes oscilaciones estacionarias de la termoclina - conocidas en limnología como secas internas - así como corrientes baroclínicas de fondo, debidas al calentamiento diferencial de distintas partes del lago.

La importancia de ambos fenómenos reside en que tanto las corrientes baroclínicas como las secas internas pueden influir en el estado trófico del lago. Así, las corrientes baroclínicas que se des-

Limnetica, 7: 23-36 (1991)

(C) Asociación Española de Limnología, Madrid. Spain plazan por encima del fondo hacen posible que un mayor volumen de agua esté en contacto directo con el sedimento, a la vez que el rozamiento entre capas de agua distintas favorece la mezcla y fuerza el transporte vertical (LEMIN \& IMBODEN, 1987). Además, el desplazamiento del agua desde zonas poco profundas a otras considerablemente más profundas puede favorecer, por ejemplo, el transporte de fitoplancton desde una zona iluminada a otra menos iluminada, pero más rica en nutrientes y predadores (Goldman \& HoKne, 1983).

Por otra parte el desplazamiento vertical de las secas produce un movimiento de ida y vuelta del agua profunda por encima del sedimento y favorece también la mezcla de substancias presentes en el sedimento (Powell \& Jassby, 1974). Además los desplazamientos horizontales durante los períodos de secas pueden afectar, por ejemplo, la distribución especial de colonias de fitoplancton (Webster, 1990). Finalmente, cabe considerar que las corrientes baroclínicas N-S y las contra- 
corrientes que deben generarse en sentido inverso tienden a reducir las diferencias entre los dos lóbulos del lago (ROBERTSOn \& RAGOTZKIE, 1990).

El lago de Banyoles se encuentra en Catalunya, en la comarca del Pla de l'Estany. Tiene una superficie de $111,19 \mathrm{hm}$ ' y un volumen de 13,12 hm' (Moreno \& Garcia-Bertiou, 1989). Su ori-

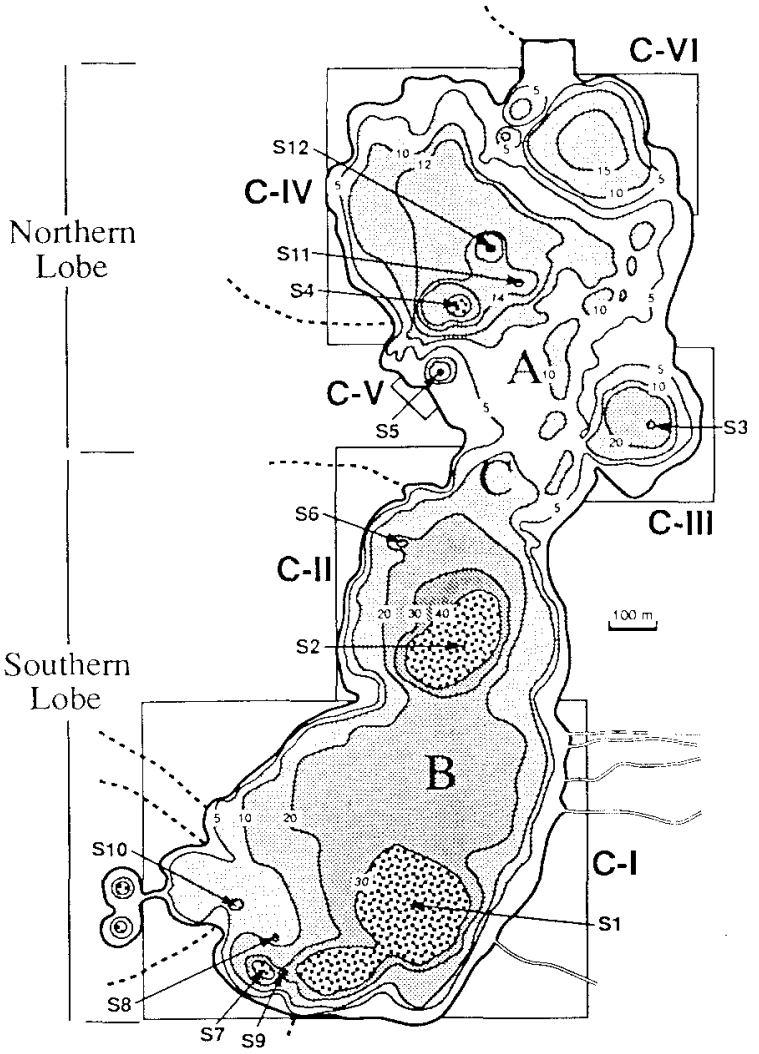

Figura 1.- Mapa hatiinéirico: el Lago de Banyoles está formado por seis cubetas principales (CI-CVI) y 12 surgencias (S1-S12). Visto desde la superficie se suele distinguir entre el Ióbulo sur (cubetas I y II) y el Ióbulo norte. La trama más gruesa indica zonas de surgencia con lodo. Las letras A, B y C muestran los puntos de donde se tomaron los datos presentados en este artículo, y a los cuales se hace referencia en el texto. (Dibujo de D. Brusi o partir de MORENo \& GARCía-BERTHOU. 1989).

Bathymetric map: Lake Banyoles has six inain basins (from CI to $\mathrm{CVI}$ ) and 12 underground springs (from S1 to S12). However. from surface, the lake secms to be composed by two lobes, the southern lobe (CI and CII) and thc northen lobe. In the shaded arcas the incoming groundwatcr keeps sediment in suspension. The letters $\mathrm{A}, \mathrm{B}$ and $C$ show the location where data presented in this article were taken. (Drawn by D. Brusi from MORFNO \& GARCIA-BFRTHOU. 1989) gen es cárstico y se alimenta por su fondo a través de distintas zonas surgentes que se han formado a partir de colapsos del terreno. Eso hace que, a pesar de su tamaño relativamente pequeño, tenga una morfometría peculiar y esté formado por diferentes cubetas. agrupadas en lo que llamaremos el Ióbulo norte (formado por las cubetas CIII, CIV, CV y CVI), y el lóbulo sur (formado por la CII y la CI) (fig. 1). Las distintas características morfológicas de cada cubeta propician que el agua se mezcle de forma distinta en cada una de ellas (Casamitiana \& Roget, 1990). Sin embargo, el agua en los primeros $20 \mathrm{~m}$ de las cubetas I y II (lóbulo sur) se mezcla uniformemente. El volumen de agua en las cubetas CI y CII es el $71 \%$ del volumen total, por lo cual nos referiremos a ellos cuando hablemos de la evolución de la mezcla del lago.

\section{MATERIAL Y MÉTODOS}

Los datos experimentales que se presentan en este artículo han sido obtenidos con diversos instrumentos Aanderaa. Estos son: una cadena de 11 termistores con una sensibilidad de $0,03{ }^{\circ} \mathrm{C}$ y una precisión de $0,05^{\circ} \mathrm{C}$; un correntímetro cuyo nivel inferior de registro es de $1,4 \mathrm{cmls}$ y cuya sensibilidad es de $0,1 \mathrm{cmls}$ para el módulo de la velocidad y de $5^{\circ}$ para la dirección; un sensor de temperatura incorporado al correntímetro anterior con las mismas características que los de la cadena de termistores, y finalmente, los registradores de velocidad y dirección del viento de una estación meteorológica situada a $100 \mathrm{~m}$ de la orilla este. a $12 \mathrm{~m}$ de altura. El límite inferior de registro para la veleta corresponde a una velocidad de $0,3 \mathrm{~m} / \mathrm{s}$, su sensibilidad es de $5^{\circ}$ y la imprecisión de otros $5^{\circ}$; la sensibilidad del anemómetro es de $0,1 \mathrm{~m} / \mathrm{s}$.

La localización de los puntos de muestreo (A, B y C) se indican en la figura 1.

Para la discusión teórica de las secas internas usaremos el modelo de Defant para secas superficiales, recogido y modificado por Mortimer para secas internas (Mortimer, 1979). Se trata de un modelo unidimensional y iterativo de integración de las ecuaciones de continuidad y de movimiento. 


\section{RESULTADOS}

\section{Afloramientos y secas}

En la figura 2 se presenta la temperatura del agua cada 20 minutos a 5, 7, 8, 10, 13 y $19 \mathrm{~m}$, en el Ióbulo sur, durante un período de mezcla. El 28 de septiembre la termoclina estacional se hallaba aproximadamente a siete metros de profundidad. Un mes más tarde, el 28 de octubre, los 20 m superiores de la columna de agua del Ióbulo sur estaban mezclados. En dicha figura también podemos observar como a 8 metros la mezcla se realizó de forma claramente abrupta. Esta coincidió con fuertes vientos que forzaron el afloramiento de agua hipolimnética en el extremo del lago situado en la dirección de donde procedía el viento; posteriormente aparecieron secas internas.

En la figura 3 podemos observar, en grupos de diez días, los vientos registrados durante el mes de octubre de 1989. La velocidad representada es la máxima para intervalos de 20 minutos. Observamos que predominan los vientos de dirección norte (alrededor de los $330^{\circ}$ ) y sureste (alrededor de los 120"). También se observa que los vientos de mayor velocidad tienen una fuerte componente norte. La velocidad media, no presentada aquí, tiene un comportamiento totalmente análogo, siendo los valores máximos de unos $5 \mathrm{~m} / \mathrm{s}$. El hecho de que el eje longitudinal del lago siga la di-

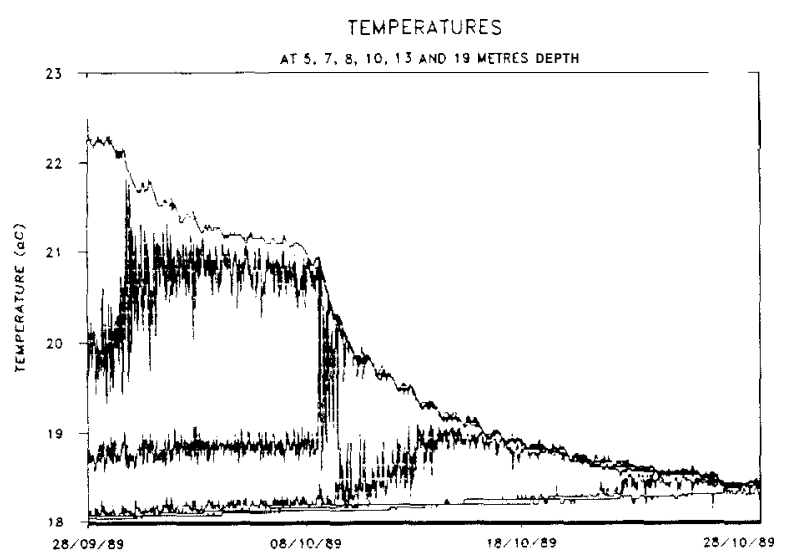

Figura 2.- Evolución de la temperatura a distintas profundidades, durante el periodo de mezcla del 1989.

Evolution of the temperature at different depths, during the mixing period in 1989 .

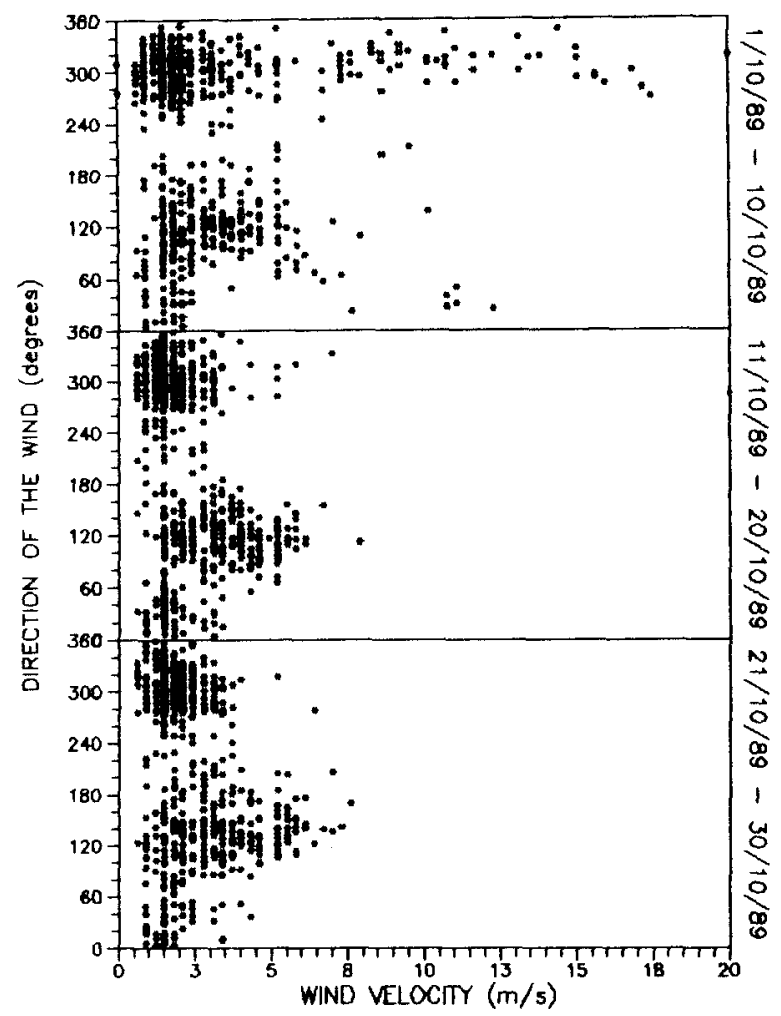

Figura 3.- Velocidad máxima del viento. cada 20 minutos, en función de su dirección. durante el mes de octubre de 1989. Maximum wind velocity, every 20 minutes. related to its direction, during October 1989.

rección de los vientos dominantes, esto es la dirección norte-sur, favorece la excitación de las secas (STOCKER et al., 1987).

En la figura 4a se representa la temperatura en el Ióbulo sur (B), en la zona de la termoclina, a 8 m de profundidad. así como la velocidad registrada por el correntímetro - situado por encima de la termoclina, en la parte sur del lóbulo norte (C), y la velocidad del viento, promediada cada veinte minutos, durante el 8 de octubre de 1989. En dicha figura puede observarse como en el minuto 220 , correspondiente a las 13 h 22 m del día 89109108, se registró un viento fuerte. Dos horas después la temperatura registrada por la cadena a $8 \mathrm{~m}$ de profundidad había aumentado dos grados. Mientras tanto, el correntímetro registraba velocidades comprendidas entre $1,5 \mathrm{~cm} / \mathrm{s}$ y $4 \mathrm{cmls}$.

En la figura $4 \mathrm{~b}$ se representa la dirección de donde procedía el viento durante el mismo perío- 
do y la dirección hacia donde iba la corriente, según los convenios aceptados en meteorología y oceanografía, respectivamente. Vemos pues que durante este período el viento procedía del norte, mientras que la corriente iba hacia el norte, es decir, en la dirección contraria.

Esta fenomenología es la característica de los afloramientos (fig. 5a): un punto A situado inicialmente por debajo de la termoclina en la parte del lago hacia donde sopla el viento pasa a estar por encima de ésta, ya que la termoclina se inclina debido al aumento de presión creado por el transporte del agua. Sin embargo, esta situación es inestable y el agua vuelve a donde procede. La diferencia de densidad entre el agua de encima de la termoclina - más caliente y por tanto menos densa- y la de debajo, hace que una resbale sobre la otra. Así se produce una corriente neta so-

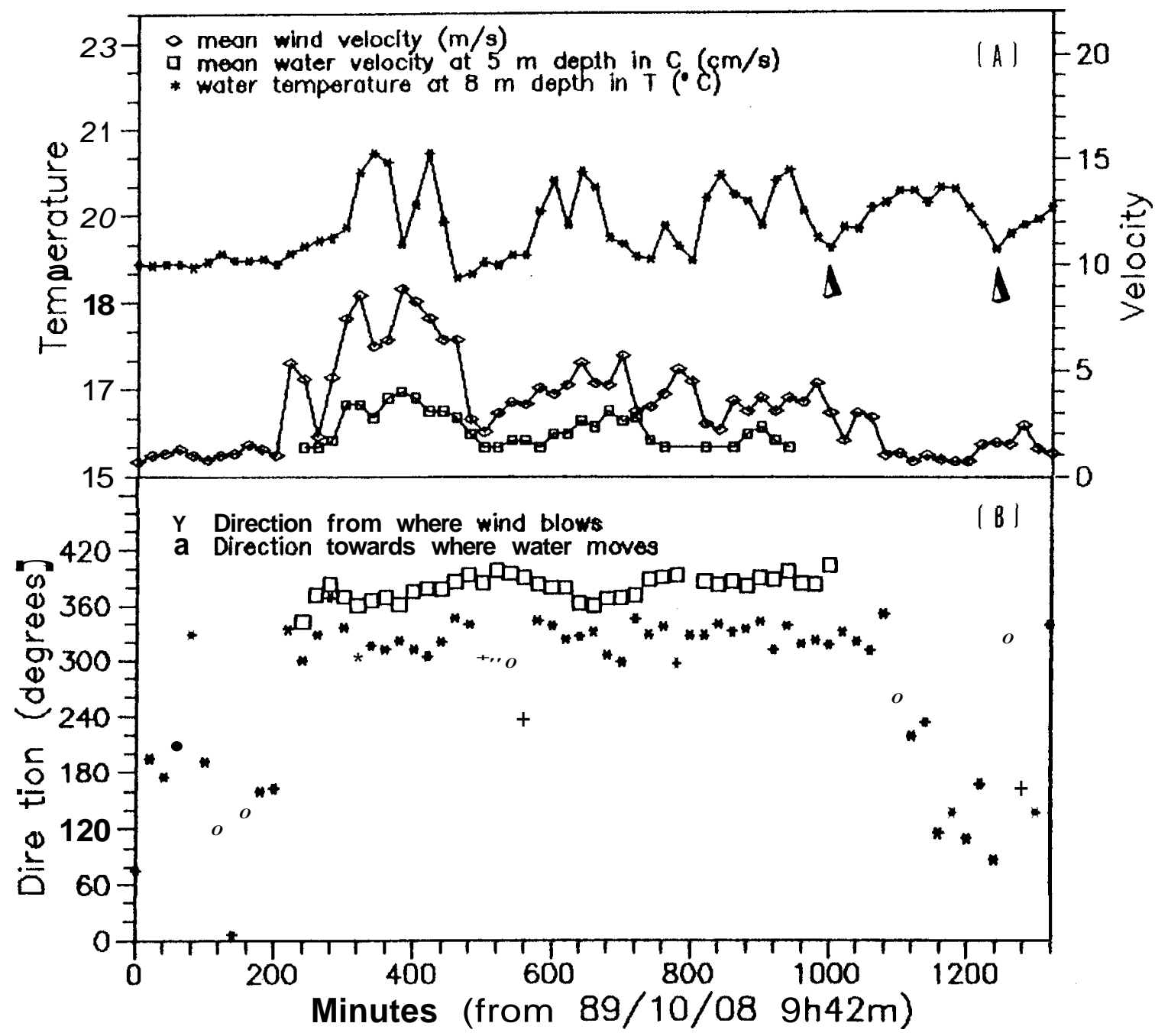

Figura 4.- (a): Observación de un afloramiento de la termoclina a partir de los datos de la temperatura en una profundidad que inicialmente se encontraba por encima de la termoclina, y de la corriente por encima de la interfase, correlacionados con el viento. (h): Direcciones opuestas del viento y el correntímetro situado por encima de la termoclina, durante el afloramiento.

(a): Observation of a set up of the thermoclinc from temperature data taken at a depth which at the begining was above the thermocline and from water velocity above the interface, correlated to the wind. (b): Opposited dircctions of the wind and the water over the thermoclinc. during the sct up. 
bre la termoclina que va en dirección contraria a la que tiene el agua arrastrada directamente por el viento.

Volviendo a la figura 4, vemos que después de amainar el viento fuerte, alrededor del minuto 1000, hay aún una fluctuación de la temperatura de más de un grado (los extremos de dicha fluctuación se representan en la figura con triángulos). La duración de ésta - unas cuatro horas-se corresponde, como veremos más adelante, con el primer modo propio de oscilación de la termoclina para el lóbulo sur, en aquellas condiciones de estratificación. De hecho. cabría esperar que

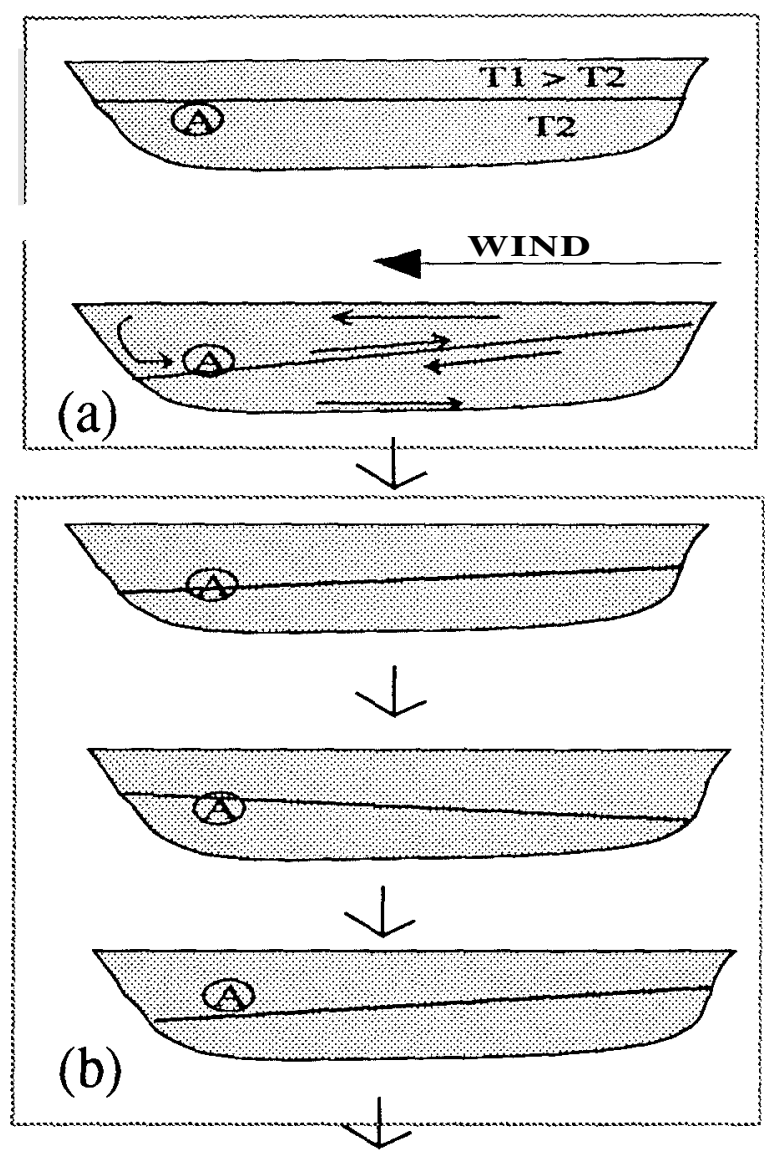

Figura 5.- Resumen de la fenomenología del afloramiento (5a) y el subsiguiente movimiento oscilatorio de la interfase - secas internas - una vez el viento ya ha amainado (5b).

Schematic draw of a set up (5a) and of the consequent internal seiches when the wind stops (5b) después de la oscilación observada hubiera habido nuevas oscilaciones cada vez con una amplitud menor hasta volver al equilibrio (fig. 5 b). Sin embargo. en este caso la calma duró poco y el viento actuó de nuevo forzando otro afloramiento. Pero la segunda vez, cuando el viento volvió a amainar sí que se detectaron estas oscilaciones, tal como puede verse en la figura 6 .

En la figura 6a, durante los aproximadamente 900 primeros minutos, se observa la misma fenomenología del afloramiento comentada en la figura 4a. Seguidamente, cuando la intensidad del viento disminuye (alrededor del minuto 900), el correntímetro deja de marcar y aparecen, a $9 \mathrm{~m}$ de profundidad, oscilaciones de la temperatura con un período del orden de 4 h 30' que siguen durante casi todo el día. El correntímetro, situado en la zona con menor sección transversal —con la idea de poder registrar el desplazamiento del agua durante el afloramiento - coincide aproximadamente con un punto nodal, como se verá después; por lo tanto, cuando ya se han excitado las secas internas, no registra velocidad ninguna. En la figura $6 \mathrm{~b}$ se representan las direcciones del viento y la corriente, según los mismos convenios utilizados en la figura 4b. También en este caso, durante el afloramiento el viento procede del norte. mientras que el agua a $5 \mathrm{~m}$ de profundidad, en el punto $\mathrm{C}$, va del sur hacia el norte.

\section{Corrientes baroclínicas}

En la figura 7 se presenta la evolución de la temperatura a 13 y a $19 \mathrm{~m}$ en el lóbulo sur (B) durante el mes de noviembre de 1989. Observamos como durante los primeros días la temperatura a 13 y a 19 m es prácticamente la misma. Poco después. a $19 \mathrm{~m}$ (uno por encima del fondo), aparece agua ligeramente más fría que la que se encuentra a $13 \mathrm{~m}$ y que presenta variaciones máximas de temperatura de unos $0,4{ }^{\circ} \mathrm{C}$. Observamos que a 13 metros no se dan variaciones de este orden.

Por otra parte, en la figura 8 se representa, durante el mismo período, la evolución de la temperatura en el Ióbulo norte (A) a $5 \mathrm{~m}$ de profundidad y en el Ióbulo sur (B) a 19 m. A partir del 4 de noviembre se constata un claro paralelismo entre el comportamiento a $5 \mathrm{~m}$ en el lóbulo norte y a 19 m en el sur. En las dos series de temperatura representadas en dicha figura se observa un ritmo 
diario. A $5 \mathrm{~m}$ de profundidad estas variaciones responden a la alternancia díainoche. Sin embargo, las variaciones observadas a $19 \mathrm{~m}$ de profundidad no pueden deberse a la misma causa ya que a 13 m estas variaciones no son observadas (fig. 7).

En la figura 9 se presenta la velocidad (módulo y dirección) de corriente registrada a 16 metros de profundidad — uno por encima del fondo- en la parte central del lago, en noviembre del 1990. La componente NIS del viento también se representa en la figura 9. Las condiciones meteorológicas durante este período fueron suaves y no presentan ningún suceso importante, excepto en los días 10, $16,25,26$ y 30 , en los cuales la velocidad media diaria del viento fueron superiores a 1,5 mis. Exceptuando estos días, se observa que la corriente

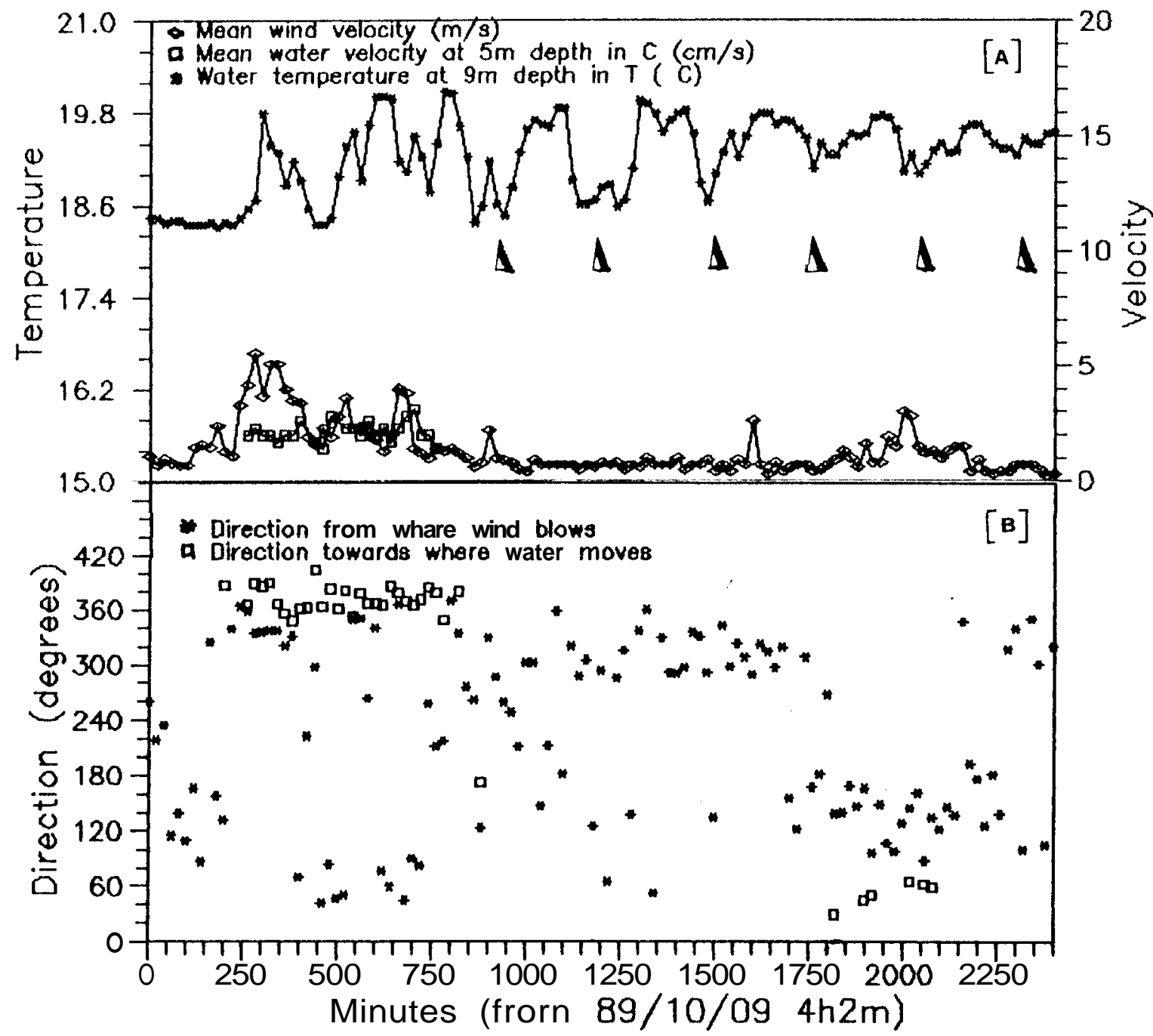

Figura 6.- (a): Nuevo afloramiento observado a partir de los datos de la temperatura en una profundidad que inicialmente se encontraba por encima de la terrnoclina y de la corriente por encima de la interfase, correlacionados con el viento. (b): Observación, durante un afloramiento, de las direcciones opuestas del viento y el agua — cuando la sensibilidad del correntímetro lo permite por encima dc la termoclina, y las subsiguientes secas internas (triángulos).

(a) New set up. observed from temperature data taken at a depth which at the begining was above the thermocline and from water velocity above the interface, correlated to the wind. (b) Opposited directions of the wind and of the water - when the sensivity of the currentmeter is enough - above the thermocline, during a set up and the consequent internal seiches (triangles). 
Southern lobs: 13 and $19 \mathrm{~m}$. depth

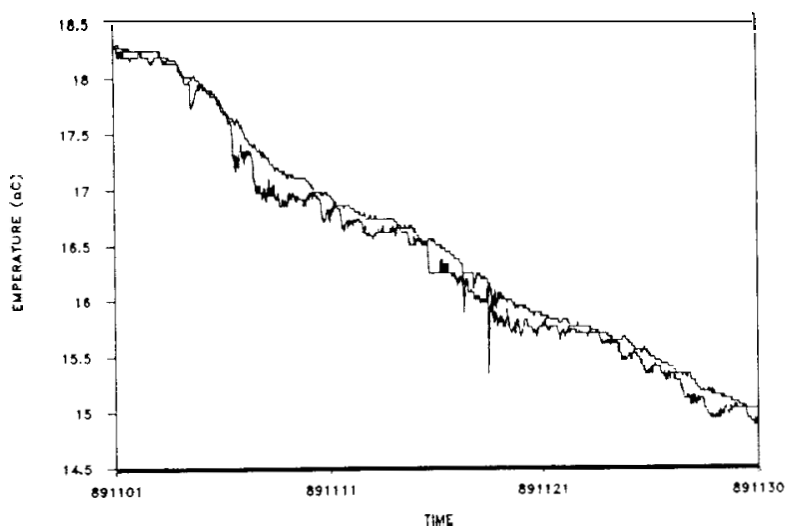

Figura 7.- Evolución de la temperatura a 13 y 19 metros de profundidad en el lóbulo sur. Contrasta el comportamiento suave a los 13 metros con las intrusiones de agua más fría a 19 metros. Temperature evolution at 13 and 19 metrcs depth in the southern lobe. The smooth behaviour at 13 metres contrasts with the colder water intrusions at 19 metrcs depth.

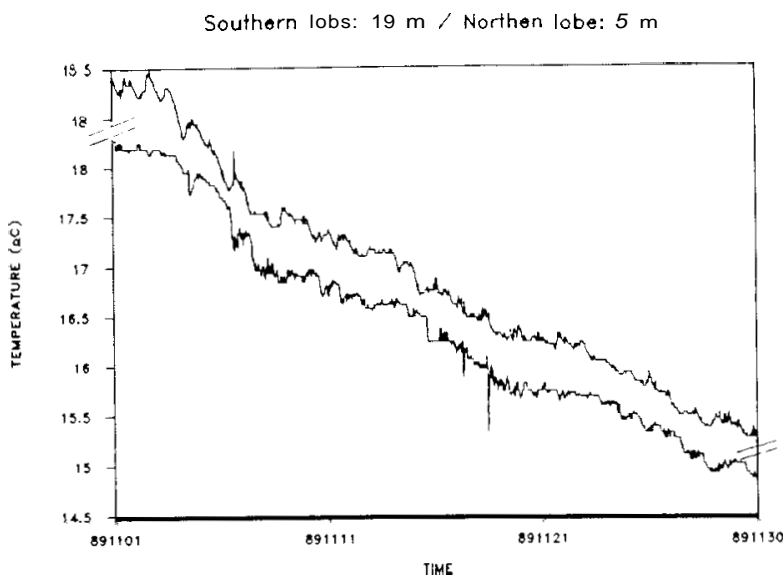

Figura 8.- Se observa la analogía existente cntre la evolución de la temperatura a 5 metros en el lóbulo norte y a 19 metros en el sur.

An analogous behaviour at 5 mctres depth in the northern lobe and at 19 metres depth in the southern is observed.
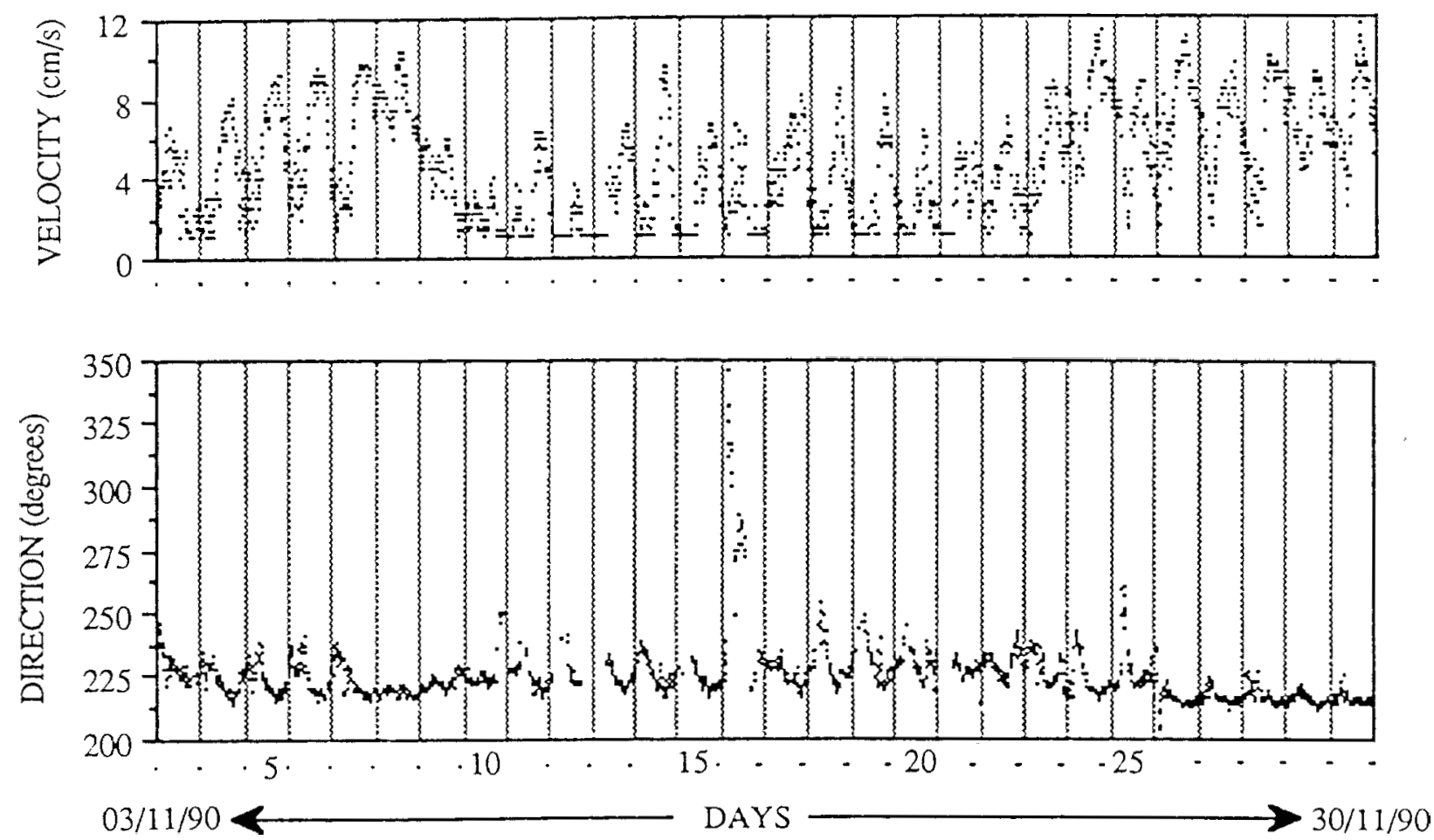

Figura 9.- Velocidad y dirección de la corriente baroclínica, registradas en la parte central del lago (C). 1 metro por encima del fondo y la componente norte/sur del viento.

Velocity and direction of the baroclinic cdrrent in location $\mathrm{C}$, in the central part of the lake, 1 metre above the bottom and the North/South component of the wind. 


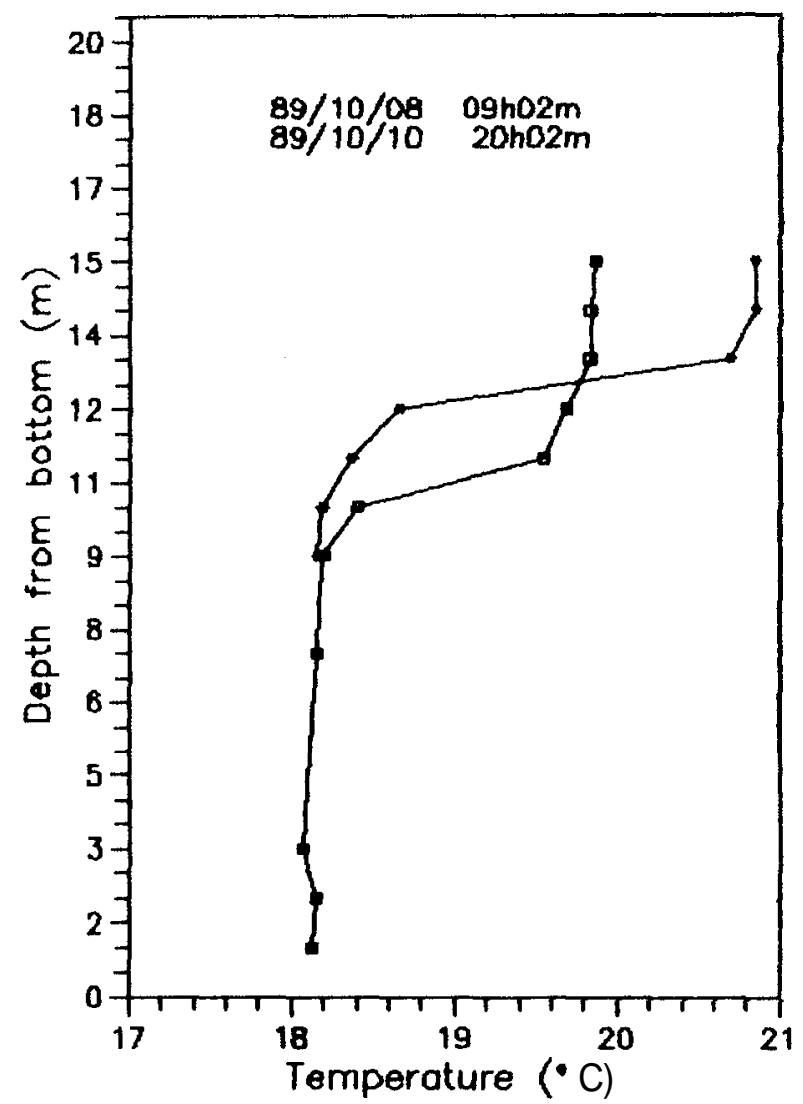

Figura 10.- Perfiles verticales de temperatura antes y después de los afloramientos y de la excitación de los modos propios de oscilación de la terrnoclina.

Vertical temperature profiles before and after the set up and the internal sciches.

tiene siempre una componente neta importante en la dirección sur-sur, aunque esté ligeramente desviada hacia el sureste.

\section{DISCUSIÓN}

\section{Afloramientos y secas}

En la figura 10 se representan los perfiles verticales de temperatura antes y después de los períodos de afloramientos y secas representados en las figuras 4 y 6 . Antes de dicho período, a $9 \mathrm{~m}$ de profundidad, se observa un gradiente de $2{ }^{\circ} \mathrm{C}$ por $\mathrm{m}$. A pesar del afloramiento representado en la figura 4 , dicho gradiente no había variado cuando sucedió el nuevo afloramiento y las consiguientes secas internas (fig. 6). Dichas secas presentan variaciones de la temperatura de unos $2{ }^{\circ} \mathrm{C}$, por lo que, según el gradiente observado, se deduce que en el lugar de la medida, hubo una variación del nivel de la termoclina de $1 \mathrm{~m}$. Más adelante veremos como, según el modelo de Defant/Mortimer, esto implica que en el extremo del lago hubo un afloramiento de unos dos metros.

De la figura 6a se deduce que el período de las secas observadas es aproximadamente de $4 \mathrm{~h} 40$ m. La precisión, debida al intervalo de medida es de 20 minutos. Para las condiciones de estratificación en este caso (esto es $\Delta \mathrm{Q}=0,000375 \mathrm{gr} / \mathrm{cm}^{3}$ ) el modelo de Defant/Mortimer predice para el segundo modo de oscilación del lago, un período de 4 h 23 m. La excitación del segundo modo es usual en lagos que, como el de Banyoles, presentan un estrechamiento central que permite considerar el lago como la unión de dos cubetas (ver por ejemplo, STOCKER et al., 1987). Esta característica morfológica Fuerza la existencia de un nodo en el cuello de unión entre los dos lóbulos, de manera que el segundo modo del conjunto del lago coincide con el primero de los lóbulos por separado. En el caso presentado, no se disponen de datos del lóbulo norte y por lo tanto desconocemos si estaba o no excitado. Sin embargo, cuando durante el octubre siguiente se registró en el lóbulo sur una fenomenología totalmente análoga a la aquí presentada, se dispuso de otra cadena de termistores en el lóbulo norte y no se registraron secas internas. Por lo tanto parece plausible afirmar que las secas internas en el lóbulo norte se excitan, en todo caso, raramente.

Los desplazamientos verticales y horizontales de la termoclina en las secciones transversales escogidas, obtenidos con el modelo de Defant/Mortimer, están representados en la figura 11. Estas secciones son equidistantes entre ellas $(100 \mathrm{~m}) \mathrm{y}$ se hallan sobre el eje longitudinal del lago en la dirección sur-norte. Los desplazamientos verticales observados en nuestro caso, como se ha señalado anteriormente, fueron de $1 \mathrm{~m}$. Dichos desplazamientos se midieron en la zona correspondiente a la sección transversal número 5 . De la figura 11 se deduce que los desplazamientos ver- 
STANDING INTERNAL WAVE

First mode of the Southern lobe
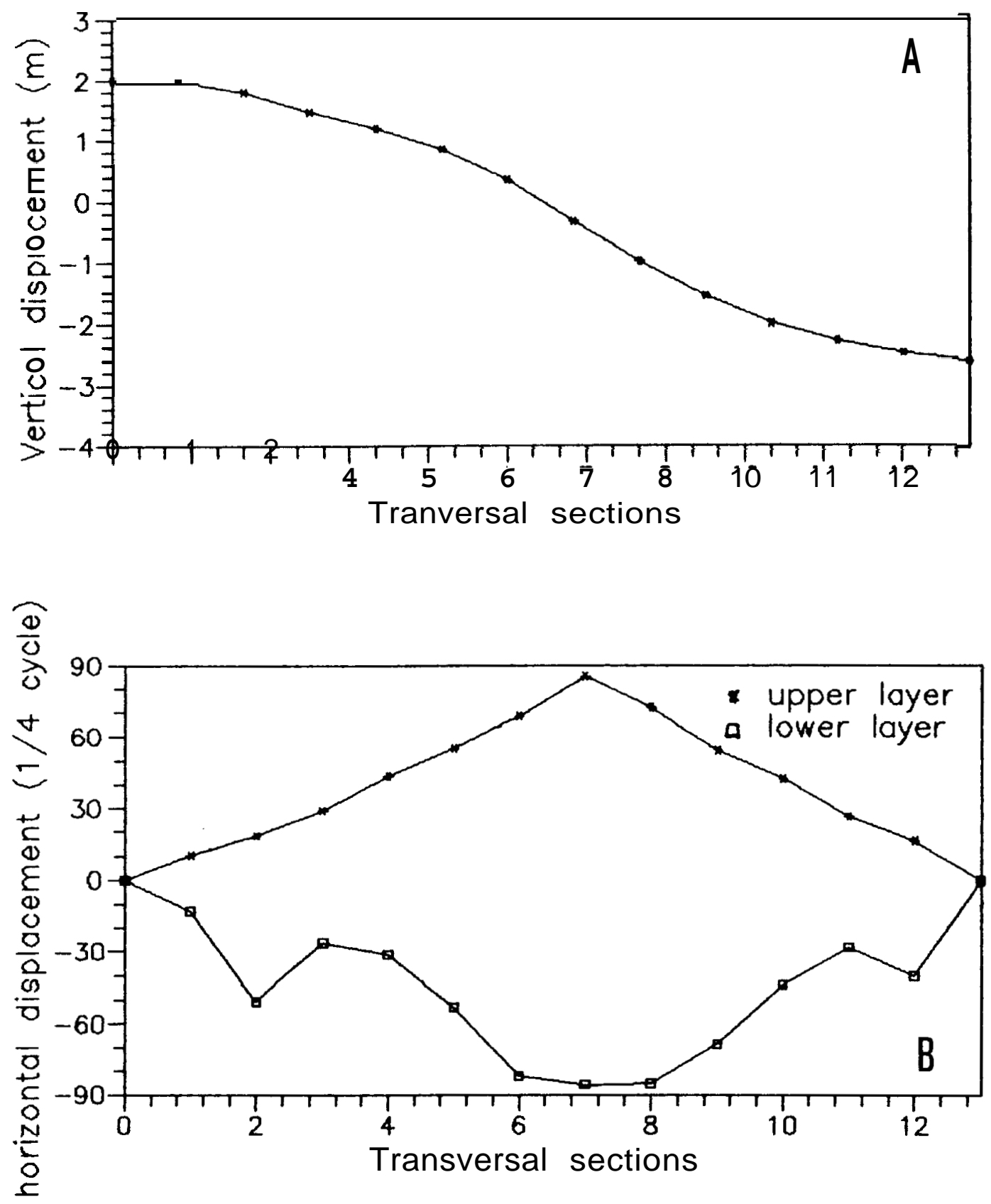

Figura 11.- Desplazamientos verticales (a) y horizontales (b) de la termoclina, obtenidos teóricamente por el método de Defant/Mortimer para el lóbulo sur, en las condiciones de estratificación reales durante las secas observadas el 10110189. Se ha supuesto un afloramiento de 2 metros y las secciones transversales se tomaron equidistantes entre ellas, siguiendo el eje longitudinal del lóbulo en la dirección sur-norte.

Vertical (a) and horizontal (b) displacements obtained theoretically by Mortimer/Defant method applied to the southern lobe, in the real stratification conditions observed during the seiches recordcred in 10110189 . We have suposed a set up of 2 metcrs. The transversal sections have been taken every 100 meters frorn south to north over the longitudinal axis of the lake. 
ticales en la sección 0 son el doble que en la 5 . Por lo tanto, en el caso estudiado, el afloramiento en el extremo del lago (sección 0) fué del orden de 2 metros.

En la figura 12 se presentan otros resultados que el modelo de Defant/Mortimer predice. En la parte superior se representan las velocidades medias y máximas en las mismas secciones, para este caso. La velocidad máxima esperada es de unos $3 \mathrm{~cm} / \mathrm{s}$, alrededor de la sección 7 , situada $700 \mathrm{~m}$ hacia el norte a partir de la ribera sur. En la figura $12 \mathrm{~b}$ se presenta el caudal desplazado en medio ciclo, el cual, en la parte central del lóbulo sur, es de unos $300.000 \mathrm{~m}$ '.

A partir de una evaluación de las escalas de las magnitudes características del lago, del estado de estratificación del lago y de la corriente de agua existente, se puede calcular el número de Richard-

\section{STANDING INTERNAL WAVE \\ First mode of the Southern lobe}

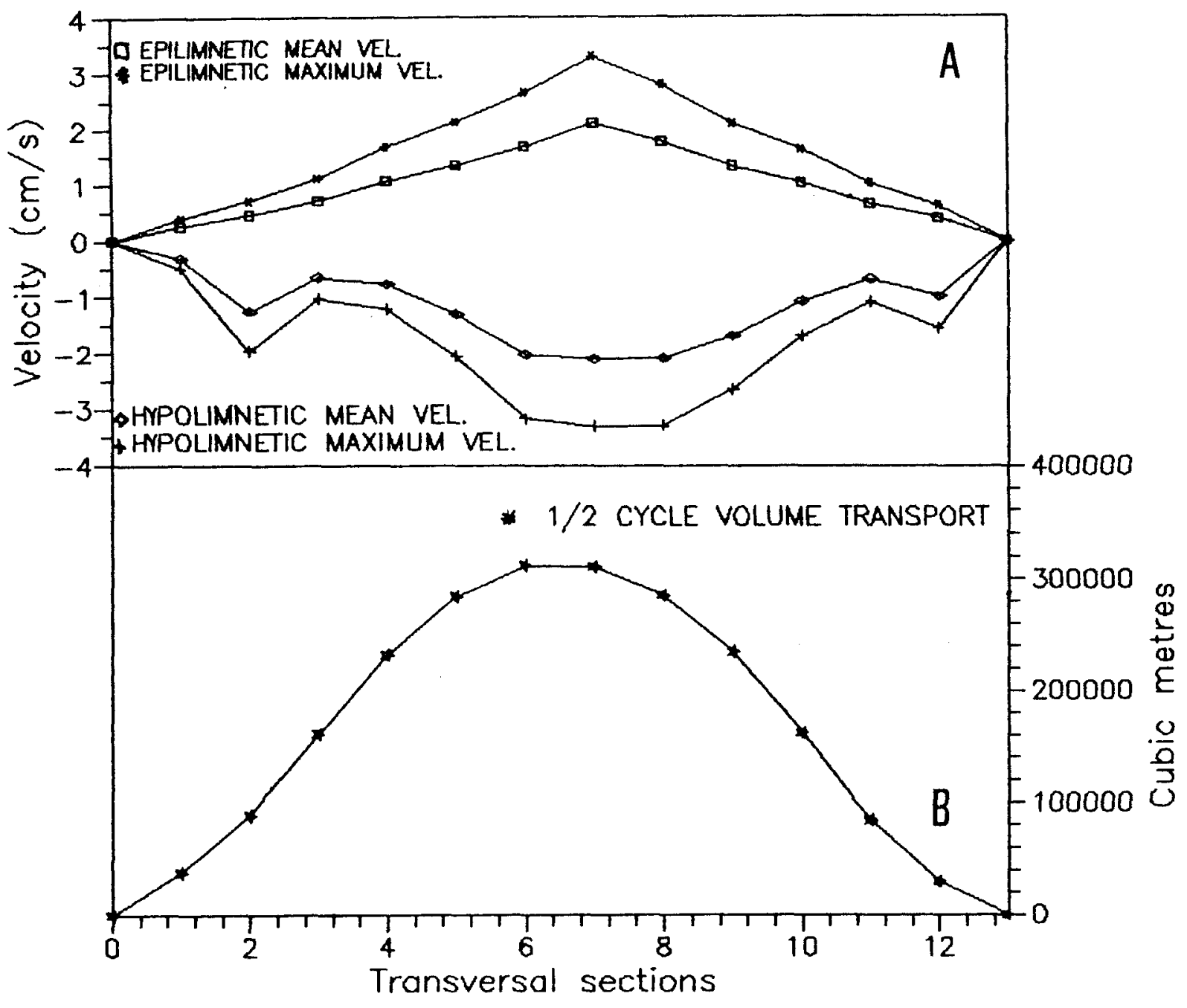

Figura 12.- Velocidades máximas y medias en el hipolimnion y en el epilimnion (a) y caudales desplazados (b) obtenidos por el método de Defant/Mortimer para las secas internas observadas el 10/10/89.

Maximum and mcan water velocity in the hypolimnion and in the epilimnion (a) and volume flows (b) obtained by Defant/Mortimer method applied to the internal seiches observed in 10110189. 
son $\left(R i=\alpha g \Delta T h / u^{2}\right)$. Éste es un parámetro adimensional que está relacionado directamente con la estabilidad del sistema, y a partir del cual puede efectuarse una primera evaluación del tipo de régimen de mezcla que caracteriza al período de secas (SPIEgel \& IMBERGer, 1980). En el numerador del número de Richardson tenemos la estabilidad en la interfase debida a la estratificación, mientras que en el denominador hay el elemento desestabilizador, en este caso la velocidad del agua debida al efecto del viento. En el episodio representado en la figura 6 , hallamos que $(\mathrm{L} / 2 \mathrm{~h})<\mathrm{Ri}<(\mathrm{L} / 2 \mathrm{~h})^{2}$, donde $\mathrm{h}$ es la profundidad a que se halla la termoclina y L la longitud del lago en la dirección que sopla el viento; en nuestro caso L coincide con el eje longitudinal del lago. En estas condiciones, las ondas internas son características. Los afloramientos y rizamientos de la termoclina no son significativos, desde el punto de vista de su importancia en la mezcla, aunque la amplitud de las oscilaciones puede ser considerable. La erosión mantiene la interfase muy abrupta y localizada. La energía de mezcla proviene principalmente del efecto de mezcla causado directamente por el viento (FISHER et al. 1979).

\section{Corrientes baroclínicas}

De las figuras 7 y 8, y considerando el hecho de que en invierno el Ióbulo norte está ligeramente más frío que el Ióbulo sur, se puede deducir que el agua más fría, y por tanto más densa, del lóbulo norte, pasó a ocupar una situación inestable y se hundió mientras se desplazaba hacia el sur.

La diferencia de temperatura entre los dos lobulos se debe a la diferente profundidad media de ambos lóbulos, lo cual hace que presenten diferente inercia térmica. Además este efecto viene reforzado por el aporte calorífico asociado a su alimentación subterránea. Dicho flujo calorífico es mayor (alrededor del $90 \%$ del total) en el Ióbulo sur que en el norte. Esta diferencia (unos $2 \mathrm{~W} / \mathrm{m}^{2}$ ) es mayor en invierno debido a que el flujo calorífico total (unos $16 \mathrm{~W} / \mathrm{m}^{2}$ ) también es mayor $\left(\mathrm{C}_{\mathrm{A}}\right.$ SAMITJANA et al., 1988).

Por otra parte, a pesar que en el Ióbulo norte hay aportes de agua (cubetas surgentes con aportación subterránea y riachuelos) las acequias de salida del agua están todas en el lóbulo sur. Así por ejemplo, en noviembre de 1987, el aporte sub- terráneo en el Ióbulo norte era de $30+10$ l/s, y el de los riachuelos de $100 \pm 10 \mathrm{l} / \mathrm{s}$. Esto da un caudal total de $130 \pm 20$ lis que se desplaza del norte hacia el sur (RogEt, 1987). Este caudal representa un $20 \%$ de los aportes totales al lago, y puede favorecer el establecimiento de corrientes baroclínicas, las cuales se desarrollaran debido a las condiciones térmicas diferenciales entre los dos lóbulos y a su diferente profundidad.

Sin embargo, los aportes en el Ióbulo norte no explican la magnitud de las corrientes observadas en la parte central del lago ya que, por ejemplo, si consideramos el caudal neto de 150 lis medido en noviembre, y la velocidad media diaria observada en la figura 9 durante la misma época (unos $3 \mathrm{cmis}$ ) esto implicaría que esta corriente debería tener una sección transversal de sólo $3 \mathrm{~m}^{2}$. Sin embargo, esta corriente es detectada en una amplia área transversal. Por lo tanto, si bien la tendencia neta es que el agua se desplace de norte a sur, la necesidad de desagüe del lóbulo norte no explica la magnitud de estas corrientes, las cuales son debidas a las inestabilidades baroclínicas. Así pues, fuera de su situación de equilibrio, el agua del lóbulo norte - más pesada - se hunde y desciende por la pendiente del fondo entre los dos lóbulos desviándose hacia la ribera oeste hasta llegar al lóbulo sur. De la figura 9 se deduce que dicha corriente se refuerza cuando el viento del $\mathrm{S}$ desplaza el agua hacia el $\mathrm{N}$ y aumenta la presión en dicho Ióbulo.

El hecho de que la corriente se desvíe más hacia el oeste cuando menor es su velocidad (fig. 9) podría explicarse por el efecto de Coriolis el cual induce círculos de inercia menores a menores velocidades. El número de Rossby (Pedlosky, 1987) es un parámetro adimensional que relaciona el tiempo característico del fenómeno estudiado con el período inercial debido al efecto de Coriolis. Por otra parte, otro número adimensional, el radio de Rossby, compara las longitudes características de ambos procesos. En nuestro caso, obtenemos para el número de Rossby local valores extremos de 0.3 y 0,1 , lo cual apunta a que el efecto de Coriolis puede ser perceptible en el comportamiento de estas corrientes. Esto, a su vez, es compatible con los radios de Rossby obtenidos (de 368 y $153 \mathrm{~m}$ ) ya que estos son menores, bien que del mismo orden, que la mínima distancia entre costas opuestas. que es de unos 500 metros. En am- 
bos cálculos cabe considerar que los valores obtenidos representan un límite superior ya que en ambos números adimensionales la velocidad aparece en el numerador y los valores de la velocidad utilizados han sido los representados en la figura 9, los cuales corresponden a la sección transversal menor del lago y por tanto se trata de velocidades máximas.

Las dos intrusiones puntuales de agua aún más fría (entre los días 11 y 21) que se detectan a 19 m en el lóbulo sur (fig. 7 y 8), se explican por una llegada puntual (durante más de 2 horas) de agua más fría procedente de la cubeta II, que por sus características morfométricas se mezcla con dificultad a partir de los $20 \mathrm{~m}$ (CAsamitjana \& RoGET, 1990).

\section{AGRADECIMIENTOS}

Los autores desean agradecer la colaboración de J. Colomer, quien ha participado en los muestreos de forma regular y en la planificación de las campañas de medidas. Un primer tratamiento sistemático de los datos obtenidos ha sido realizado por A. Costa y M. Quintana. Este proyecto ha sido parcialmente subvencionado con una ayuda AR89 concedida por la CIRIT de la Generalitat de Catalunya, a uno de los autores.

\section{Bibliografía}

Casamitjana, X. \& E. Rogiet, 1990. The thermal structure of Lake Banyoles. Verh. Internat. Verein. Limnol., 24: 88-91.

Casamitjana, X., E. Rogit, D. Jou \& E. Llebot, 1988. Effect of suspended sediment on the heating of Lake Banyoles. Journal of Geophysical Research. C8, (93), 9332-9336

FisHeR, H.B., E.J. LIST, R.C.Y. KoH, J. IMBERGER \& N.H BRoOKs, 1979. Mixing in inland and coastal waters. Academic Press, Inc., 483 pgs.

Goldman \& Horne, 1983. Limnology. McGraw-Hill

Lemmin, U. \& D.H. Imboden, 1987. Dynamics of bottom currents in a small lake. Limnol. Oceunogr., 32 (1): 62-75.

Moreno-Amich. R. \& E. Garcia-Berthou, 1989. A new bathymetric map based on echo-sounding and morphometrical characterization of the Lake of Banyoles (NE-Spain). Hydrobiologia, 185: 83-90.

Mortimer, C.H., 1979. Strategies for coupling data collection and analysis with dynamic modelling of lake motion. Lake hydrodynamics, p. 183-277. Symp. Proc. Elsevier.

Pedlosky, J., 1987. Gcophysical Fluid Dynamics. 2nd edition. Springer-Verlag, $710 \mathrm{pp}$.

ROGET, E., Estudio de los caudales surgentes por las cubetas de Banyolcs. 1987. (en catalán). Tesina de Licenciatura. Universidad Autónoma de Barcelona.

Robertson, A.M. \& R.A. Ragotzkie, 1990. Thermal structure of a multibasin lake: influence of morphometry, interbasin exchange. and groundwater. Can. J. Fish. Aquat. Sci. 47: 1206-1212

SPigel. R.H. \& J. Imberger, 1980. The classification of mixed-layer dynamics in lakes of small to medium size. Jour nal of Physical Oceunogruphy, 40: 1104-1121.

Stocker. K., K. Hutter, G. Salvadé, J. Trösch, F. ZamBONI, 1987. Observations and analysis of internal seiches in the southern basin of Lake Lugano. Annales Geophysicue, 5B, (6), 553-568.

WEBSTER, I.J., 1990. Effect of wind on the distribution of phytoplankton cells in lakes. Limnol. Oceanogr., 35 (5), 989-1001. 